Pacific Journal of Mathematics

INTEGRAL BASES FOR BICYCLIC BIQUADRATIC FIELDS Jar John Parry 


\title{
INTEGRAL BASES FOR BICYCLIC BIQUADRATIC FIELDS OVER QUADRATIC SUBFIELDS
}

\author{
Robert H. Bird and Charles J. Parry
}

\begin{abstract}
Explicit conditions are given for a bicyclic biquadratic number field to have an integral basis over a quadratic subfield.
\end{abstract}

A classical question of algebraic number theory is, "When does an algebraic number field $K$ have an integral basis over a subfield $k$ ?"

A complete and explicit answer to the above question is given here when $K$ is a bicyclic biquadratic number field and $k$ is a quadratic subfield. Moreover, an explicit integral basis is given for $K / k$ whenever one exists. In the cases where $k$ is imaginary or $k$ is real and has a unit of norm -1 , the conditions involve only rational congruences. When $k$ is real and the fundamental unit of $\epsilon$ has norm +1 , the conditions sometimes involve $\epsilon$.

1. Notation and preliminary remarks. Throughout this article the following notation shall be used:

$Q$ : field of rational numbers.

$Z$ : rational integers.

$m, n$ : square free integers.

$l=(m, n) \geq 0, m=m_{1} l, n=n_{1} l$ and $d=m_{1} n_{1}$.

$K=Q(\sqrt{m}, \sqrt{n}):$ bicyclic biquadratic field.

$k=Q(\sqrt{m})$.

$\delta_{L / M}$ : different of an extension $L / M$.

$N(\epsilon)$ : norm of the unit $\epsilon$.

$p, q$ : odd prime numbers.

An integral basis for $K$ over $Q$ has been determined in [1, 3, 6]. Here an integral basis for $K$ over $k=Q(\sqrt{m})$ will be determined whenever it exists. In these considerations the roles of $n$ and $d$ are interchangeable so it will only be necessary to consider seven pairs of congruence classes for $(m, n)$ modulo 4 ; namely $(1,1),(1,2),(1,3),(2,1)$, $(2,3),(3,1)$ and $(3,2)$.

It follows immediately from [5] that $K$ has an integral basis over $k$ if and only if $K=k\left(D^{\frac{1}{2}}\right)$ where $(D)$ is the discriminant of $K$ over $k$. Since $K$ is a quadratic extension of $k$ the discriminant is the square of the different $\delta$. In $[3,6]$ the different of $K$ over $Q$ is explicitly determined by: 


$$
\delta_{K / Q}^{2}=\left\{\begin{array}{l}
\left(\operatorname{lm}_{1} n_{1}\right) \text { when }(m, n) \equiv(1,1)(\bmod 4) . \\
\left(4 \operatorname{lm}_{1} n_{1}\right) \text { when exactly one of } m \text { and } n \text { is } 1(\bmod 4) . \\
\left(8 \operatorname{lm}_{1} n_{1}\right) \text { when }(m, n) \text { is }(2,3) \text { or }(3,2)(\bmod 4) .
\end{array}\right.
$$

Since $\delta_{K / Q}=\delta_{K / k} \cdot \delta_{k / Q}$ and $\delta_{k / Q}=(\sqrt{m})$ or $(2 \sqrt{m})$ according as $m \equiv$ $1(\bmod 4)$ or not, the following useful result is obtained:

Lemma I. The different $\delta=\delta_{\mathrm{K} / \mathrm{k}}$ is determined (and hence the discriminant) by:

$$
\delta^{2}=\left\{\begin{array}{l}
\left(n_{1}\right) \text { when } n \equiv 1(\bmod 4) . \\
\left(4 n_{1}\right) \text { when } m \equiv 1 \text { and } n \neq \equiv 1(\bmod 4) . \\
\left(2 n_{1}\right) \text { when } m \neq 1 \text { and } n \neq \equiv 1(\bmod 4) .
\end{array}\right.
$$

2. Imaginary subfield $k$. Although some of our results here will also apply to the real case we shall be primarily concerned with the case where $k$ is an imaginary quadratic field. The main result of this section is:

THEOREM I. If $k=Q(\sqrt{m})$ is an imaginary quadratic field then $K$ has an integral basis over $k$ if and only if one of the following conditions hold:

(a) At least one of $m$ or $n$ is $1(\bmod 4)$ and $l=1$ or $-m$.

(b) $(m, n) \equiv(2,3)(\bmod 4)$ and $m=-2 l$.

(c) $m=-1$.

Furthermore, when an integral basis exists, it can be determined by the following table:

TABLE I

\begin{tabular}{lll}
\hline Basis & $(m, n)(\bmod 4)$ & Conditions \\
\hline $1,(1+\sqrt{n}) / 2$ & $(, 1)$ & $l=1$ \\
$1,(\sqrt{m}+\sqrt{d}) / 2$ & $(, 1)$ & $l= \pm m$ \\
$1, \sqrt{ \pm n_{1}}$ & $(1, n), n \neq 1(\bmod 4)$ & $l=1$ or $\pm m$ \\
$1,(\sqrt{m}+\sqrt{d}) / 2$ & $(2,3)$ & $l= \pm m / 2$. \\
$1,(\sqrt{n}+\sqrt{-n}) / 2$ & $(3,2)$ & $m=-1$
\end{tabular}

The proof will follow from a series of lemmas. First, even when $m$ is positive, it is easily seen that the conditions of Theorem I are sufficient for the existence of an integral basis. 
LEMMA II. Whenever the conditions of any line of Table I are fulfilled, even when $m$ is positive, then $K$ has the stated integral basis over $k$.

Proof. In each case it is a simple matter to check that the given basis is a basis of integers with discriminant equal to that given by Lemma I.

Our attention will now be directed to proving that the conditions of Theorem I are necessary for the existence of an integral basis when $m$ is negative.

LEMMA III. If $m$ is negative and at least one of $m$ or $n$ is $1(\bmod 4)$ then an integral basis exists if and only if $l=1$ or $-m$.

Proof. From Lemma I and Mann's criteria the existence of an integral basis is seen to be equivalent to the condition

$$
K=k\left(\sqrt{\epsilon n_{1}}\right)
$$

where $\epsilon$ is a unit of $k$. When $m \neq-1$ or -3 the only units of $k$ are \pm 1 so the above condition implies that $Q\left(\sqrt{ \pm n_{1}}\right)$ is a quadratic subfield of $K$. Thus $n_{1}=n=\ln _{1}$ or $-n_{1}=d=m_{1} n_{1}$, so either $l=1$ or $l=-m$. If $m=-1$ or -3 then $l=(n, m)$ must necessarily be 1 or $-m$.

Lemma IV. If $m$ is negative and $(m, n) \equiv(2,3)(\bmod 4)$ then an integral basis exists if and only if $m=-2 l$.

Proof. Here Mann's criteria is equivalent to

$$
K=k\left(\sqrt{ \pm 2 n_{1}}\right)
$$

so that $Q\left(\sqrt{ \pm 2 n_{1}}\right)$ is a quadratic subfield of $K$. Since $n \equiv 3(\bmod 4)$ this implies that $d=m_{1} n_{1}= \pm 2 n_{1}$ so that $m_{1}= \pm 2$. Since $m$ is negative $m_{1}=-2$ and so $m=-2 l$.

Lemma $V$. When $m$ is negative and $(m, n) \equiv(3,2)(\bmod 4)$ then an integral basis exists if and only if $m=-1$.

Proof. Again Mann's criteria gives

$$
K=k\left(\sqrt{2 \epsilon n_{1}}\right)
$$


with $\epsilon$ a unit of $k$. When $m \neq-1$ then $\epsilon= \pm 1$ so $Q\left(\sqrt{ \pm 2 n_{1}}\right)$ is again a quadratic subfield of $K$. Thus $l=2$ or $m_{1}=-2$ both of which are impossible with $m \equiv 3(\bmod 4)$. Hence $K$ has no integral basis over $k$ unless $m=-1$.

The next result is a stronger version of Theorem 4 of [5] for our special case.

COROLlaRY I. If $m$ is negative then $k$ has odd class number if and only if $K=k(\sqrt{n})$ has an integral basis over $k$ for every square free integer $n$.

Proof. It is well known that $k$ has odd class number if and only if $m=-1,-2$ or $-p$ with $p \equiv 3(\bmod 4)$. If $m$ is one of these values it is immediate from Theorem I that an integral basis exists. Conversely if $m$ has two distinct prime divisors $p$ and $p^{\prime}$ then it follows from Theorem I that $K=k(\sqrt{a p})$ has no integral basis over $k$ when $a$ is integer satisfying $(a, m)=1$ and $a p \equiv 1(\bmod 4)$. Finally if $m=-p$ with $p \equiv 1(\bmod 4)$ then $m \equiv 3(\bmod 4)$ so no integral basis exists for any $n \equiv 2(\bmod 4)$.

3. Real subfield $k$. When $k$ is a real subfield it follows from Mann's criteria and Lemma I that $K$ will have an integral basis over $k$ if and only if $K=k\left(\sqrt{2^{e} \epsilon n_{1}}\right)$ where $e=0$ or 1 and $\epsilon$ is a unit of $k$. Now every unit $\epsilon$ of $k$ has the form $\epsilon= \pm \epsilon_{0}^{j}$ where $\epsilon_{0}$ is a fundamental unit and $j$ is an integer, For any field $k$ it is easily seen that $\epsilon_{0}^{3}=b_{0}+c_{0} \sqrt{m}$ with $b_{0}, c_{0} \in Z$. Since only the parity of $j$ is important we shall assume that $j=0,1$ or 3 with the latter choice being made to insure that $\epsilon=b+c \sqrt{m}$ with $b, c \in Z$. Furthermore when $\epsilon_{0}$ has norm -1 it is easily seen that $j=0$ and whenever $j=0$ the conditions of Theorem I are necessary and sufficient for $K$ to have an integral basis over $k$.

From now on we shall only be concerned with fields $k$ where $\epsilon_{0}$ and hence $\epsilon$ has norm +1 . The following results on units will be very useful.

LEMMA VI. Let $\epsilon=\epsilon_{0}$ or $\epsilon_{0}^{3}$ have the form $b+c \sqrt{m}$ with $b, c \in Z$ and let the norm of $\epsilon$ be +1 . If $m \equiv 1$ or $2(\bmod 4)$ then $(b, c) \equiv$ $(1,0)(\bmod 2)$ and $c \equiv 0(\bmod 4)$ whenever $m \equiv 1(\bmod 4)$. Furthermore

$$
\sqrt{\epsilon}=s \sqrt{u}+t \sqrt{v}
$$

with $(u, v)=1$ and $u v=m$. If $m \equiv 3(\bmod 4)$ then either $c \equiv$ $0(\bmod 4)$ and equation $(1)$ holds or $(b, c) \equiv(0,1)(\bmod 2)$ and 


$$
\sqrt{\epsilon}=\frac{s \sqrt{2 u}+t \sqrt{2 v}}{2}
$$

with the above conditions on $u$ and $v$.

Proof. The congruence conditions are easy to verify. By [4]

$$
\begin{aligned}
\sqrt{\epsilon} & =\frac{\sqrt{N(\epsilon+1)}+\sqrt{-N(\epsilon-1)}}{2} \\
& =\frac{\sqrt{2(b+1)}+\sqrt{2(b-1)}}{2} .
\end{aligned}
$$

When $b$ is odd set $4 s^{2} u=2(b+1)$ and $4 t^{2} v=2(b-1)$ with $u$ and $v$ square free. It is easily seen that $(u, v)=1$. Also $c^{2} m=b^{2}-1=$ $4 s^{2} t^{2} u v$ so $u v=m$. When $b$ is even set $s^{2} u=b+1$ and $t^{2} v=b-1$ with $u$ and $v$ square free. As above $(u, v)=1$ and $u v=m$.

Our main objective of this section is to prove the following result:

THEOREM II. If $k=Q(\sqrt{m})$ is a real quadratic field then $K$ has an integral basis over $k$ if and only if one of the following conditions hold:

(a) At least one of $m, n$ is $1(\bmod 4)$ and either $l=1, m, u$, or $v$ with $u$ and $v$ determined by equation (1).

(b) $(m, n) \equiv(2,3)(\bmod 4)$ and $2 l=m$, u or $v$.

(c) $(m, n) \equiv(3,2)(\bmod 4)$ and $l=u$ or $v$ where $u$ and $v$ are determined by equation (2).

Furthermore, when $l=1, m / 2$ or $m$ an integral basis is given by Table $I$ and when $l=u, v, u / 2, v / 2$ an integral basis is given by Table II below. For this table we set $\sqrt{\epsilon}=(s \sqrt{r u}+t \sqrt{r u}) / r$ where $r=1$ or 2. Unless otherwise stated it will be assumed that $r=1$ and $l=u$ or $v$.

TABLE II

\begin{tabular}{cll}
\hline Basis & $(m, n)(\bmod 4)$ & Conditions \\
\hline $1,\left(1+\sqrt{\epsilon n_{1}}\right) / 2$ & $(, 1)$ & $b n_{1} \equiv 1, c \equiv 0(\bmod 4)$ \\
$1,\left(\sqrt{m}+\sqrt{\epsilon n_{1}}\right) / 2$ & $(3,1)$ & $b n_{1} \equiv 3, c \equiv 0(\bmod 4)$ \\
$1,\left(1+\sqrt{m}+\sqrt{\epsilon n_{1}}\right) / 2$ & $(2,1)$ & $b n_{1} \equiv 3, c \equiv 2(\bmod 4)$ \\
$1, \sqrt{\epsilon n_{1}}$ & $(1,3)$ or $(1,2)$ & \\
$1, \sqrt{2 \epsilon n_{1}} / 2$ & $(3,2)$ & $r=2$ \\
$1,\left(\sqrt{m}+\sqrt{2 \epsilon n_{1}}\right) / 2$ & $(2,3)$ & $2 l=u$ or $v$
\end{tabular}

Proof. In our preliminary remarks it was observed that we need only consider fields $K$ satisfying $K=k\left(\sqrt{2^{e} \epsilon n_{1}}\right)$ where $\epsilon=\epsilon_{0}^{j}(j=1$ or 3$)$ 
has norm +1 . When one of $m$ or $n$ is $1(\bmod 4)$ we wish to show that $K=k\left(\sqrt{\epsilon n_{1}}\right)$ exactly when $l=u$ or $v$. Since

$$
\sqrt{\epsilon n_{1}}=\frac{s \sqrt{r u n_{1}}+t \sqrt{r v n_{1}}}{r}
$$

we see that $k\left(\sqrt{\epsilon n_{1}}\right)^{\prime}=K$ if and only if $r u n_{1}=n=\ln n_{1}$ and $r v n_{1}=d=$ $m_{1} n_{1}$ or vice-versa. In the first case this reduces to $l=r u$ and $m_{1}=r v$, but $m=l m_{1}=r^{2} u v$ is square free so $r=1$ and $l=u$. Similarly in the second case $l=v$. Thus (a) is proven. According to Mann [5, p. 170] an integral basis for $K$ over $k$, when it exists, will be given by

$$
1,\left(a+\sqrt{2^{f} \epsilon n_{1}}\right) / 2
$$

where $a$ is an integer of $k$ satisfying

$$
a^{2} \equiv 2^{f} \epsilon n_{1} \equiv 2^{f}\left(b n_{1}+c n_{1} \sqrt{m}\right)(\bmod 4)
$$

and $f=0$ or 2 according as $n \equiv 1(\bmod 4)$ or not.

When $m \equiv n \equiv 1(\bmod 4), a=h+j \omega$ with $\omega=(1+\sqrt{m}) / 2$ and $h, j \in Z$. Thus (5) becomes

$$
a^{2} \equiv h^{2}+\left(\frac{m-1}{4}\right) j^{2}+\left(2 h j+j^{2}\right) \omega \equiv b n_{1}(\bmod 4)
$$

with the last congruence following from Lemma VI. Thus $j \equiv$ $0(\bmod 2)$ and $b n_{1} \equiv h^{2} \equiv 1(\bmod 4)$ since $b n_{1}$ is odd. Thus we take $a=1$ here and an integral basis is given by the first line of Table II. When $m \neq 1$ and $n \equiv 1(\bmod 4)$ then $a=h+j \sqrt{m}$ so

$$
a^{2}=h^{2}+j^{2} m+2 h j \sqrt{m} \equiv b n_{1}+c n_{1} \sqrt{m}(\bmod 4) .
$$

Thus $c \equiv 0$ and $b \equiv 1(\bmod 2) . \quad$ When $c \equiv 0(\bmod 4)$ congruence $(7)$ reduces to

$$
h^{2}+j^{2} m \equiv b n_{1}, 2 h j \equiv 0(\bmod 4) \text {. }
$$

Either $j \equiv 0(\bmod 2)$ and $b n_{1} \equiv h^{2} \equiv 1(\bmod 4)$ or $j \equiv 1, \quad h \equiv$ $0(\bmod 2)$ so $b n_{1} \equiv j^{2} m \equiv m \equiv 3(\bmod 4)$. The last congruence holds because $b n_{1}$ is odd and $m \neq \equiv 1(\bmod 4)$. Thus when $c \equiv 0(\bmod 4)$ an integral basis is given by one of the first two lines of Table II. When $c \equiv 2(\bmod 4)(7)$ becomes 


$$
h \equiv j \equiv 1(\bmod 2)
$$

and $b n_{1} \equiv h^{2}+j^{2} m \equiv 1+m \equiv 3(\bmod 4)$ with the last congruence following because $b n_{1}$ is odd. Thus $a=1+\sqrt{m}$ and an integral basis is given by the third line of Table II.

Finally when $m \equiv 1, n \neq \equiv 1(\bmod 4)$ congruence $(5)$ becomes $a^{2} \equiv$ $0(\bmod 4)$ so $a=0$ and an integral basis is given by the fourth line of Table II.

Suppose now $(m, n) \equiv(3,2)(\bmod 4)$. Here $K=k\left(\sqrt{2 \epsilon n_{1}}\right)$ is equivalent to $2 r u n_{1}=2^{2 e} l n_{1}(e=0$ or 1$)$ and $2 r v n_{1}=2^{2 f} m_{1} n_{1}(f=0$ or 1$)$ or vice versa. Thus $2^{2 e} l=2 r u$ and hence $l=u$ and $r=2$ (since both $l$ and $u$ are odd) or else $l=v$ and $r=2$. Here $\left\{1, \sqrt{2 \epsilon n_{1}} / 2\right\}$ forms an integral basis.

Finally consider the case $(m, n) \equiv(2,3)(\bmod 4)$. Here $K=$ $k\left(\sqrt{2 \epsilon n_{1}}\right)$ if and only if $2 u n_{1}=4 l n_{1}$ and $2 v n_{1}=m_{1} n_{1}$ or vice versa. Thus $2 l=u$ or $2 l=v$. Here an integral basis is given by the last line of Table II.

CoROllaRY I. If $m$ is positive, then $K=k(\sqrt{n})$ has an integral basis over $k$ for every $n$ if and only if one of the following holds:

(a) $m=2$ or $p$.

(b) $m=2 p$ or $p q$ with $p \equiv q(\bmod 4)$ and $N(\epsilon)=1$.

Proof. When $m=2$ or $p$ then $l=1$ or $m$ so it is clear from (a), (b), and (c) of Theorem II that an integral basis exists. When $m=2 p$ and $N(\epsilon)=1$ then $l=1$ or $p$ since $n$ is odd. But $\sqrt{\epsilon}=s \sqrt{2}+t \sqrt{p}$ so $u=2$ and $v=p$, thus Theorem II is satisfied. When $m=p q$ with $p \equiv$ $q(\bmod 4)$ and $N(\epsilon)=1$ then it follows from Lemma VI that $\sqrt{\epsilon}=$ $s \sqrt{p}+t \sqrt{q}$. Thus $u=p$ and $v=q$ so (a) of Theorem II is always satisfied.

To prove the converse first note that if $m$ has 3 or more odd prime divisors then there are at least 8 choices for $l$, all of which can occur for suitably chosen values of $n$. But, on the other hand, there are only 4 values of $l$ for which Theorem II is satisfied. When $m=2 p q$ there are four possible values of $l$ which can occur, namely $l=1, p, q$ or $p q$. However, it is seen from Theorem II (a) and (b) that there are less than four possible values of $l$ where an integral basis does exist. If $m=p q$ with $p \not \equiv q(\bmod 4)$ and $r=1$ then when $n$ is even no integral basis exists. If $r=2$, then no integral basis exists when $l=p$ and $n$ odd. Finally when $m=2 p$ or $p q$ with $N(\epsilon)=-1$ then if $l=p$ and $n \equiv 1(\bmod 4)$ no integral basis exists.

COROLlARY II. If $k$ has odd class number then $K=k(\sqrt{n})$ has an integral basis over $k$ for every integer $n$. 
Proof. The field $k=Q(\sqrt{m})$ has odd class number if and only if

$$
m=2, p, 2 p_{1} \text { or } p_{1} p_{2}
$$

with $p_{1} \equiv p_{2} \equiv 3(\bmod 4)$. It is easy to see that when $m$ has a prime divisor $q \equiv 3(\bmod 4)$ that $\epsilon$ has positive norm. Hence this is an immediate result of Corollary $\mathbf{I}$.

COROLLARY III. If $k$ is a quadratic number field either every bicyclic biquadratic extension field $K$ has an integral basis over $k$ or there exist infinitely many such $K$ which do (and don't) have an integral basis over $k$.

Proof. Immediate from Theorems I and II and their corollaries.

\section{REFERENCES}

1. A. Adrian Albert, The integers of normal quartic fields, Ann. Math., 31 (1930), 381-418.

2. Harvey Cohn, A Second Course in Number Theory, John Wiley \& Sons, New York, 1962.

3. Elaine Haught, Bicyclic Biquadratic Number Fields, Masters Thesis, VPI \& SU, 1972.

4. S. Kuroda, Über die Dirichletschen Körper, J. Fac. Sci. Univ. Tokyo, 4 (1943), 383-406.

5. Henry B. Mann, On integral bases, Proc. Amer. Math. Soc., 9 (1958), 167-172.

6. Kenneth S. Williams, Integers of biquadratic fields, Canad. Math. Bull., 13 (1970), 519-526.

Received April 30, 1974.

Virginia Polytechnic Institute and State University 



\section{Pacific Journal of Mathematics}

\section{Vol. 66, No. $1 \quad$ November, 1976}

Helen Elizabeth. Adams, Factorization-prime ideals in integral domains ............ Patrick Robert Ahern and Robert Bruce Schneider, The boundary behavior of Henkin's kernel.

Daniel D. Anderson, Jacob R. Matijevic and Warren Douglas Nichols, The Krull

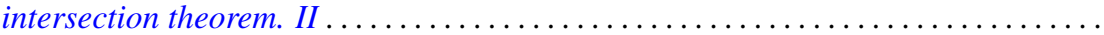

Efraim Pacillas Armendariz, On semiprime P.I.-algebras over commutative regular

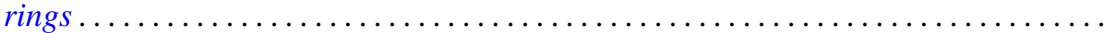

Robert H. Bird and Charles John Parry, Integral bases for bicyclic biquadratic fields

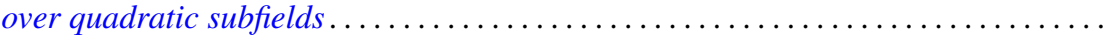

Tae Ho Choe and Young Hee Hong, Extensions of completely regular ordered

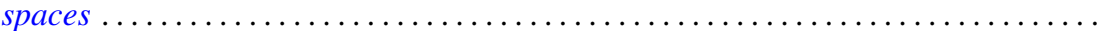

John Dauns, Generalized monoform and quasi injective modules ...............

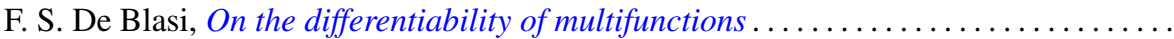

Paul M. Eakin, Jr. and Avinash Madhav Sathaye, R-endomorphisms of $R[[X]]$ are

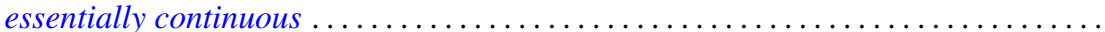

Larry Quin Eifler, Open mapping theorems for probability measures on metric

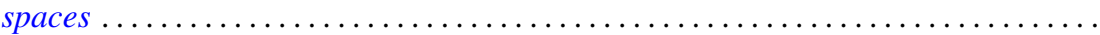

Garret J. Etgen and James Pawlowski, Oscillation criteria for second order self adjoint

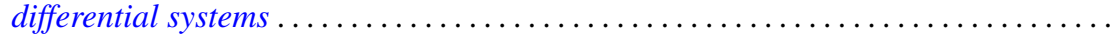

Ronald Fintushel, Local $S^{1}$ actions on 3-manifolds .

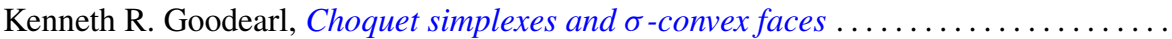

John R. Graef, Some nonoscillation criteria for higher order nonlinear differential

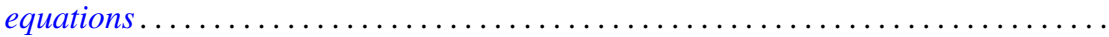

Charles Henry Heiberg, Norms of powers of absolutely convergent Fourier series: an example.

Les Andrew Karlovitz, Existence of fixed points of nonexpansive mappings in a space

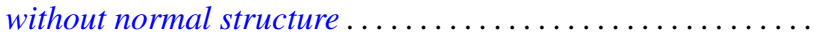

Gangaram S. Ladde, Systems of functional differential inequalities and functional

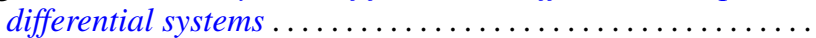

Joseph Michael Lambert, Conditions for simultaneous approximation and interpolation with norm preservation in $C[a, b]$.

Ernest Paul Lane, Insertion of a continuous function.

Robert F. Lax, Weierstrass points of products of Riemann surfaces .

Dan McCord, An estimate of the Nielsen number and an example concerning the Lefschetz fixed point theorem...

Paul Milnes and John Sydney Pym, Counterexample in the theory of continuous functions on topological groups...

Peter Johanna I. M. De Paepe, Homomorphism spaces of algebras of holomorphic functions

Judith Ann Palagallo, A representation of additive functionals on $L^{p}$-spaces,

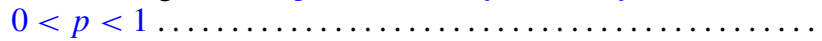

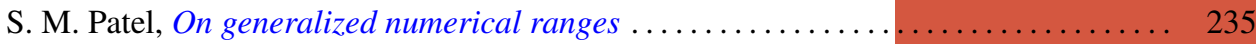

Thomas Thornton Read, A limit-point criterion for expressions with oscillatory

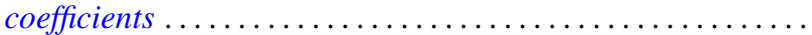

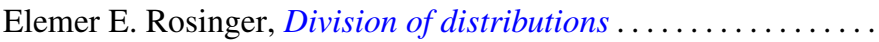

Peter S. Shoenfeld, Highly proximal and generalized almost finite

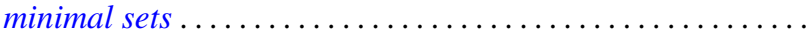

R. Sirois-Dumais and Stephen Willard, Quotient-universal sequential spaces

Robert Charles Thompson, Convex and concave functions of singular values of matrix sums....

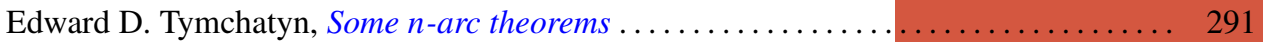

